\title{
Signatures of highly inclined accretion disks in galactic black hole candidates and AGNs
}

\author{
A. F. Zakharov ${ }^{1,2,3}$ and S. V. Repin ${ }^{4}$ \\ 1 National Astronomical Observatories, Chinese Academy of Sciences, 100012 Beijing, PR China \\ 2 Institute of Theoretical and Experimental Physics, 25, B. Cheremushkinskaya st., Moscow 117259, Russia \\ 3 Astro Space Centre of Lebedev Physics Institute, 84/32, Profsoyuznaya st., Moscow 117810, Russia \\ ${ }^{4}$ Space Research Institute, 84/32, Profsoyuznaya st., Moscow 117810, Russia
}

\begin{abstract}
Received 7 March 2003 / Accepted 18 April 2003
Abstract. Recent X-ray observations of microquasars and Seyfert galaxies reveal broad emission lines in their spectra, which can arise in the innermost parts of accretion disks. Simulations indicate that at low inclination angle the line is measured by a distant observer as characteristic two-peak profile. However, at high inclination angles $\left(>85^{\circ}\right)$ two additional peaks arise. This phenomenon was discovered by Matt et al. (1993) using the Schwarzschild black hole metric to analyze such effect. They assumed that the effect is applicable to a Kerr metric far beyond the range of parameters that they exploited. We check and confirm their hypothesis about such a structure of the spectral line shape for the Kerr metric case. We use no astrophysical assumptions about the physical structure of the emission region except the assumption that the region should be narrow enough. Positions and heights of these extra peaks drastically depend on both the radial coordinate of the emitting region (circular hot spot) and the inclination angle. We find that the best conditions to observe this effect are realized at $\theta>85^{\circ}$ and $r>5 r_{\mathrm{g}}$ and may exist in microquasars or low-mass black holes in X-ray binary systems, because there is some precession (and nutation of accretion disks) with not very long time periods (see, for example, the SS433 binary system). The line profiles for different inclination angles and radial coordinates are presented. To analyze the influence of disk models on the spectral line shapes we simulate the line profiles for the Shakura-Sunyaev disk model for accretion disks with the high inclination.
\end{abstract}

Key words. black hole physics - line: profiles - X-rays individuals: SS433

\section{Introduction}

More than ten years ago it was predicted that profiles of lines emitted by AGNs and X-ray binary systems ${ }^{1}$ could have an asymmetric double-peaked shape (e.g. Chen et al. 1989; Fabian et al. 1989; Robinson et al. 1990; Dumont \& Collin-Souffrin 1990; Matt et al. 1993). Generation of the broad $K_{\alpha}$ fluorescence lines as a result of irradiation of a cold accretion disk was discussed by many authors (see, for example, Matt et al. 1991, 1992a; Matt \& Perola 1992; Matt et al. 1993; Bao 1993; Martocchia et al. 2002 and references therein). Recent X-ray observations of Seyfert galaxies, microquasars and binary systems (Fabian et al. 1995; Tanaka et al. 1995; Nandra et al. 1997a,b; Malizia et al. 1997; Sambruna et al. 1998; Yaqoob et al. 2001; Ogle et al. 2000; Miller et al. 2002 and references therein) confirm these considerations in general and reveal broad emission lines in their spectra with characteristic

Send offprint requests to: A. F. Zakharov,

e-mail: zakharov@vitep1.itep.ru

${ }^{1}$ Some of them are microquasars (for details see, for example, Greiner 1999; Mirabel 2000; Mirabel \& Rodriguez 2002). two-peak profiles. A comprehensive review by Fabian et al. (2000) summarizes the detailed discussion of theoretical aspects of possible scenarios for generation of broad iron lines in AGNs. These lines are assumed to arise in the innermost parts of the accretion disk, where the effects of General Relativity (GR) must be taken into account, otherwise it appears very difficult to find a natural explanation for observed line profile.

Numerical simulations of the line structure are be found in a number of papers (Kojima 1991; Laor 1991; Bao \& Stuchlik 1992; Bao 1993; Bao et al. 1994; Rauch \& Blandford 1993, 1994; Bromley et al. 1997; Fanton et al. 1997; Pariev \& Bromley 1997, 1998; Pariev et al. 2001; Ruszkowski M. 2000; Ma 2002). They indicate that the accretion disks in Seyfert galaxies are usually observed at the inclination angle $\theta$ close to $30^{\circ}$ or less. This occurs because according to the Seyfert galaxy models, an opaque dusty torque surrounds the accretion disk which does not allow us to observe the disk at larger inclination angles.

However, at inclination angles $\theta>80^{\circ}$, new observational manifestations of GR could arise. (Matt et al. 1993 discovered such phenomenon for a Schwarzschild black hole, moreover 
the authors predicted that their results could be applicable to a Kerr black hole over the range of parameters exploited.) The authors mentioned that this problem was not analyzed in detail for a Kerr metric case and it would be necessary to investigate this case. Below we do not use a specific model on surface emissivity of accretion (we only assume that the emitting region is narrow enough). But general statements (which will be described below) can be generalized to a wide disk case without any problem. Therefore, in this paper we check and confirm their hypothesis for the Kerr metric case and for a Schwarzschild black hole using other assumptions about surface emissivity of accretion disks. In principle, such a phenomenon could be observed in microquasars and X-ray binary systems where there are neutron stars and black holes with stellar masses.

In Sect. 3 we discuss disk models used for simulations. In Sect. 4 we present the results of simulations with two extra peaks in the line profile. These extra peaks exist because of the gravitational lens effect in the strong field approximation of GR. In Sect. 5 we discuss results of calculations and present some conclusions.

\section{Numerical methods}

We used an approach discussed in detail in papers by Zakharov (1991, 1994), Zakharov \& Repin (1999, 20002a), Zakharov \& Repin (2002b, 2003), Zakharov et al. (2002). The approach was used in particular to simulate spectral line shapes. For example, Zakharov et al. (2002) used this approach to simulate the influence of a magnetic field on spectral line profiles. This approach is based on results of a qualitative analysis (which was done by Zakharov 1986, 1989 for different types of geodesics near a Kerr black hole). The equations of photon motion in the Kerr metric are reduced to the following system of ordinary differential equations in dimensionless Boyer-Lindquist coordinates (Zakharov 1991, 1994; Zakharov \& Repin 1999):

$$
\begin{aligned}
\frac{\mathrm{d} t}{\mathrm{~d} \sigma} & =-a\left(a \sin ^{2} \theta-\xi\right)+\frac{r^{2}+a^{2}}{\Delta}\left(r^{2}+a^{2}-\xi a\right), \\
\frac{\mathrm{d} r}{\mathrm{~d} \sigma} & =r_{1},
\end{aligned}
$$

$\frac{\mathrm{d} r_{1}}{\mathrm{~d} \sigma}=2 r^{3}+\left(a^{2}-\xi^{2}-\eta\right) r+(a-\xi)^{2}+\eta$

$\frac{\mathrm{d} \theta}{\mathrm{d} \sigma}=\theta_{1}$

$\frac{\mathrm{d} \theta_{1}}{\mathrm{~d} \sigma}=\cos \theta\left(\frac{\xi^{2}}{\sin ^{3} \theta}-a^{2} \sin \theta\right)$

$\frac{\mathrm{d} \phi}{\mathrm{d} \sigma}=-\left(a-\frac{\xi}{\sin ^{2} \theta}\right)+\frac{a}{\Delta}\left(r^{2}+a^{2}-\xi a\right)$,

where $\Delta=r^{2}-2 r+a^{2} ; \eta=Q / M^{2} E^{2}$ and $\xi=L_{z} / M E$ are the Chandrasekhar constants (Chandrasekhar 1983) which are derived from the initial conditions of the emitted quantum in the disk plane. The system (1)-(6) has two first integrals

$$
\begin{aligned}
\epsilon_{1} \equiv & r_{1}^{2}-r^{4}-\left(a^{2}-\xi^{2}-\eta\right) r^{2} \\
& -2\left[(a-\xi)^{2}+\eta\right] r+a^{2} \eta=0 \\
\epsilon_{2} \equiv & \theta_{1}^{2}-\eta-\cos ^{2} \theta\left(a^{2}-\frac{\xi^{2}}{\sin ^{2} \theta}\right)=0,
\end{aligned}
$$

which can be used for the accuracy control of computation.

Solving Eqs. (1)-(6) for monochromatic quanta emitted by a ring we can calculate a specral line shape $I_{v}(r, \theta)$ which is registered by a distant observer at inclination angle $\theta$.

The numerical integration has been performed using the combination of the Gear and Adams methods (Gear 1971) and realized as the standard package by Hindmarsh (1983), Petzold (1983), Hiebert \& Shampine (1980). We obtain the entire disk spectrum by summation of sharp ring spectra.

\section{Disk model}

To simulate the structure of the emitted line it is necessary first to choose a model for the emissivity of an accretion disk. We exploit two different models, namely we consider a narrow and thin disk moving in the equatorial plane near a Kerr black hole as the first model and as we analyze the inner wide part of an accretion disk with a temperature distribution which is chosen according to the Shakura \& Sunyaev (1973) with fixed inner and outer radii $r_{\mathrm{i}}$ and $r_{\mathrm{o}}$ as the second model. Usually a power law is used for wide disk emissivity (see, for example, Laor 1991; Matt et al. 1991; Martocchia \& Matt 1996; Martocchia et al. 2000, 2002). However, other models for emissivity can not be excluded for such a wide class of accreting black holes, therefore, to demonstrate how another emissivity law could change line profiles we investigate such a emissivity law.

First, we assume that the source of the emitting quanta is a narrow thin disk rotating in the equatorial plane of a Kerr black hole. We also assume that the disk is opaque to radiation, so that a distant observer situated on one disk side cannot measure the quanta emitted from its other side.

For the sake of computational simplicity we suggest that the spectral line is monochromatic in its co-moving frame. It can really be adopted because even at $T=10^{8} \mathrm{~K}$ the thermal line width

$\frac{\delta f}{f} \sim \frac{v}{c} \sim \frac{1}{c} \sqrt{\frac{k T}{m_{\mathrm{Fe}}}} \approx 10^{-3}$

appears to be much less than the Doppler line width associated with the Kepler velocity of the disk rotation.

For second case of the Shakura-Sunyaev disk model, we assume that the local emissivity is proportional to the surface element and and $T^{4}$, where $T$ is a local temperature. The emission intensity of the ring is proportional to its area. The area of the emitting ring (width $\mathrm{d} r$ ) differs in the Kerr metric from its classical expression $\mathrm{d} S=2 \pi r \mathrm{~d} r$ and should be replaced with

$\mathrm{d} S=\frac{2 \pi\left(r^{2}+a^{2}\right)}{\sqrt{r^{2}-r r_{\mathrm{g}}+a^{2}}} \mathrm{~d} r$. 
Thus, the total flux density emitted by the disk and registered by a distant observer is proportional to the integral

$J_{v}(\theta)=\int_{r_{\text {in }}}^{r_{\text {out }}} I_{\nu}(r, \theta) T^{4}(r) \mathrm{d} S$,

where $I_{v}(r, \theta)$ is obtained from the solution of Eqs. (1)-(6), $T(r)$ - from the appropriate dependence for a hot disk and $\mathrm{d} S-$ from Eq. (10).

For simulation purposes we assume that the emitting region lies entirely in the innermost region of the $\alpha$-disk (zone $a$ ) from $r_{\text {out }}=10 r_{\mathrm{g}}$ to $r_{\text {in }}=3 r_{\mathrm{g}}$ and the emission is monochromatic in the co-moving frame ${ }^{2}$. The frequency of this emission set as unity by convention.

\section{Simulation results}

Spectral line profiles of a narrow ring observed at large inclination angles $\theta>85^{\circ}$ and different radii $r$ are shown in Fig. 1 (a Kerr metric generalization of Fig. 1 from Matt et al. 1993 which was drawn using calculations for the Schwarzschild case). The ring is assumed to move in the equatorial plane of a Kerr black hole with an almost extreme rotation parameter $a=0.9981$. The inclination angle increases from left to right and the radial coordinate from bottom to top. The figure indicates that there are practically no new specific features of profiles, thus, the line profile remains one-peaked with a maximum close to $1.6 E_{\text {lab }}$ and a very long red wing without any significant details.

For the lowest radii there are no signatures of multiple peaks of spectral line shapes even for high inclination angles (the bottom row in Fig. 1 which corresponds to $r=0.8 r_{\mathrm{g}}$ ).

Increasing the radius to $r=1.2 r_{\mathrm{g}}$, an additional blue peak arises in the vicinity of the blue maximum at the highest inclination angle $\theta=89^{\circ}$. The red maximum is so small that no details can be distinguished in its structure. At lower inclination angles $\theta \leq 88^{\circ}$ the blue maximum also has no details and the entire line profile remains essentially one-peaked.

For $r=3 r_{\mathrm{g}}$ additional details in the blue peak appear for $\theta \geq 85^{\circ}$. Thus, for $\theta=85^{\circ}$ we have a fairly clear bump, at $\theta=88^{\circ}$ it changes into a small complementary maximum and for $\theta=89^{\circ}$ this maximum becomes well-distinguished. Its position in the last case differs significantly from the main maximum: $E_{3}=1.12 E_{\text {lab }}, E_{4}=1.34 E_{\text {lab }}$.

When further increasing the radius the red maximum also bifurcates. This effect becomes visible for $r=5 r_{\mathrm{g}}$ and $\theta \geq 88^{\circ}$. Thus, for $\theta=85^{\circ}$ we have only a faintly discernible (feebly marked) bump, but for $\theta=88^{\circ}$ both complementary maxima (red and blue) arise. For $\theta=89^{\circ}$ we have already four maxima in the line profile: $E_{1}=0.63 E_{\mathrm{lab}}, E_{2}=0.66 E_{\mathrm{lab}}, E_{3}=$ $1.07 E_{\mathrm{lab}}, E_{4}=1.28 E_{\mathrm{lab}}$. Note that the splitting for the blue and red maxima is not equal, moreover, $E_{4}-E_{3} \approx 7\left(E_{2}-E_{1}\right)$.

For $r=10 r_{\mathrm{g}}$ the profile becomes more narrow, but the complementary peaks appear very distinctive. We have a fourpeak structure for $\theta \geq 88^{\circ}$. It is interesting to note that for

\footnotetext{
${ }^{2}$ We use as usual the notation $r_{\mathrm{g}}=2 G M / c^{2}$.
}

$\theta=89^{\circ}$ the energy of the blue complementary peak is close to its laboratory value.

Note that the effect almost disappears when the radial coordinate becomes less than $r_{\mathrm{g}}$, i.e. for the orbits which could exist only near a Kerr black hole.

Figure 2 demonstrates the details of the spectrum presented in the top row in Fig. 1. Thus, the left panel includes all the quanta emitted by a hot spot at $r=10 r_{\mathrm{g}}$ with $\theta>89^{\circ}$ at infinity (the mean value could be counted as 89.5 if the quanta distribution would be uniform there) but with much higher resolution than in Fig. 1. The spectrum has four narrow distinctive maxima separated by lower emission intervals. In the right panel, which includes all the quanta with $\theta>89.5$, the right complementary maxima is even higher than the main one. The blue complementary maxima still remains lower than its main counterpart, but it increases rapidly its intensity with increasing inclination angle. It follows immediately from the comparison of the left and right panels. The "oscillation behavior" of the line profile between the maxima has a pure statistical origin and is not caused by the physics involved.

As an illustration, a spectrum of an entire accretion disk at high inclination angles in the Schwarzschild metric is shown in Fig. 3. (In reality we have calculated geodesics for the quanta trajectories in the Schwarzschild metric using the same Eqs. (1)-(6) as for a Kerr metric, but assuming there $a=0.01$.) The emitting region (from 3 to $10 r_{\mathrm{g}}$ ) lies as a whole in the innermost region of the $\alpha$-disk (the detailed description of this model was given by Shakura \& Sunyaev 1973; Lipunova \& Shakura 2002).

As follows from Fig. 3, the blue peak may consist of the two components, whereas the red one remains unresolved.

\section{Discussion and conclusions}

The complicated structure of the line profile at large inclination angles is explained by the multiple images of some pieces of the hot ring. We point out that the result was obtained in the framework of GR without any extra physical and astrophysical assumptions about the character of the radiation etc. For a Kerr black hole we assume only that the radiating ring is circular and narrow.

The problem of multiple images in the accretion disks and extra peaks was first considered by Matt et al. (1993) (see also Bao \& Stuchlik 1992; Bao 1993; Bao et al. 1994, 1996). Using numerical simulations Matt et al. (1993) proved the statement for the Schwarzschild metric and suggested that the phenomenon is applicable to a Kerr metric over the range of parameters that the authors have analyzed. They noted also that it is necessary to perform detailed calculations to confirm their hypothesis. We verified and confirmed their conjecture without any assumptions about a specific distribution of surface emissivity or accretion disk model (see Figs. 1 and 2).

We confirmed also their conclusion that extra peaks are generated by photons which are emitted by the far side of the disk, therefore we have a manifestation of gravitational lensing in the strong gravitational field approach for GR.

Some possibilities to observe considered features of spectral line profiles were considered by Matt et al. (1993), 

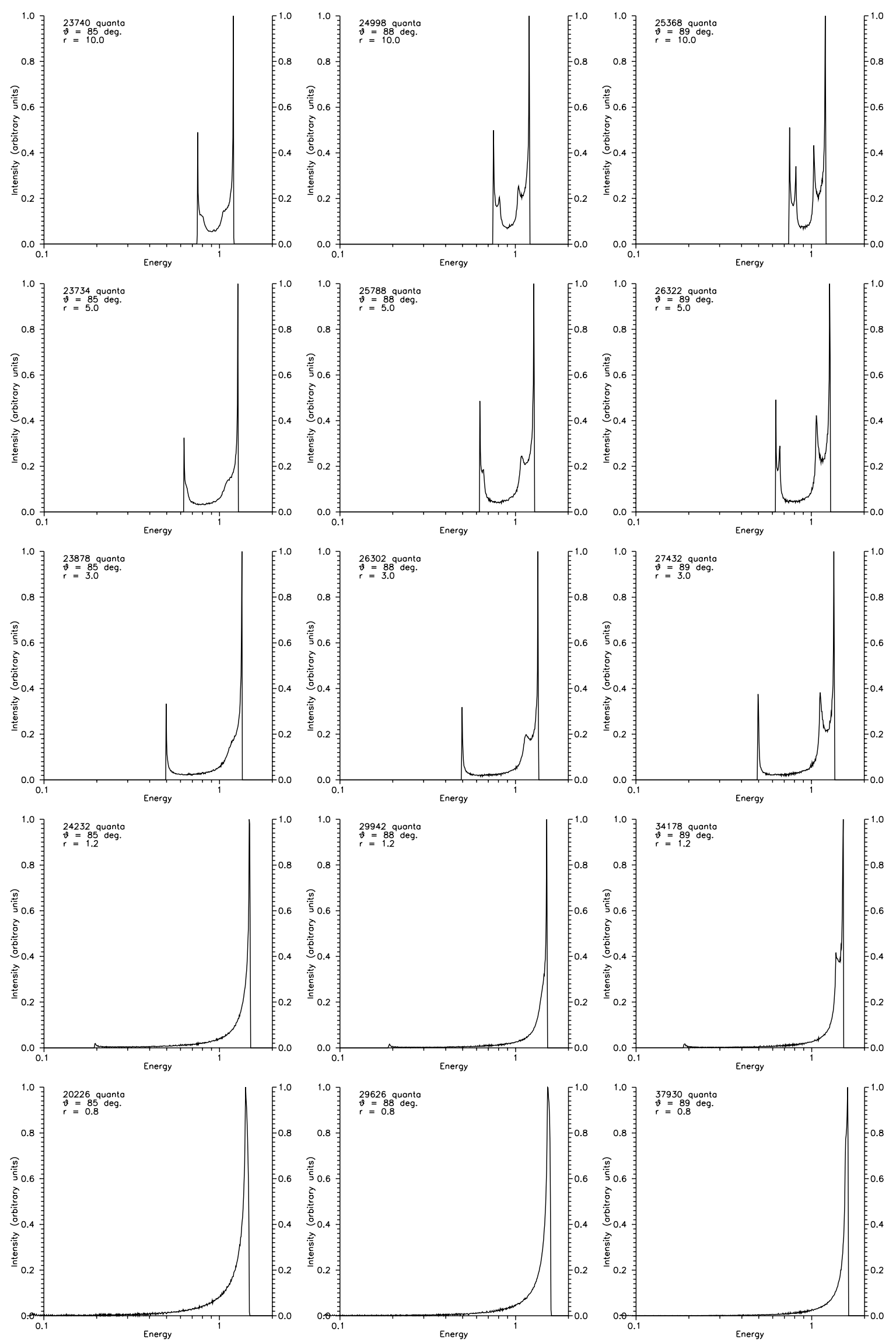

Fig. 1. Line profiles for high inclination angles, $\theta>85^{\circ}$, due to gravitational lens effects in the strong gravitational field approach. The Kerr (rotation) parameter was chosen as $a=0.9981$. The radii decrease from top to bottom, the inclination angles increase from left to right; their values are shown in each panel along with the number of quanta included in the spectrum. 

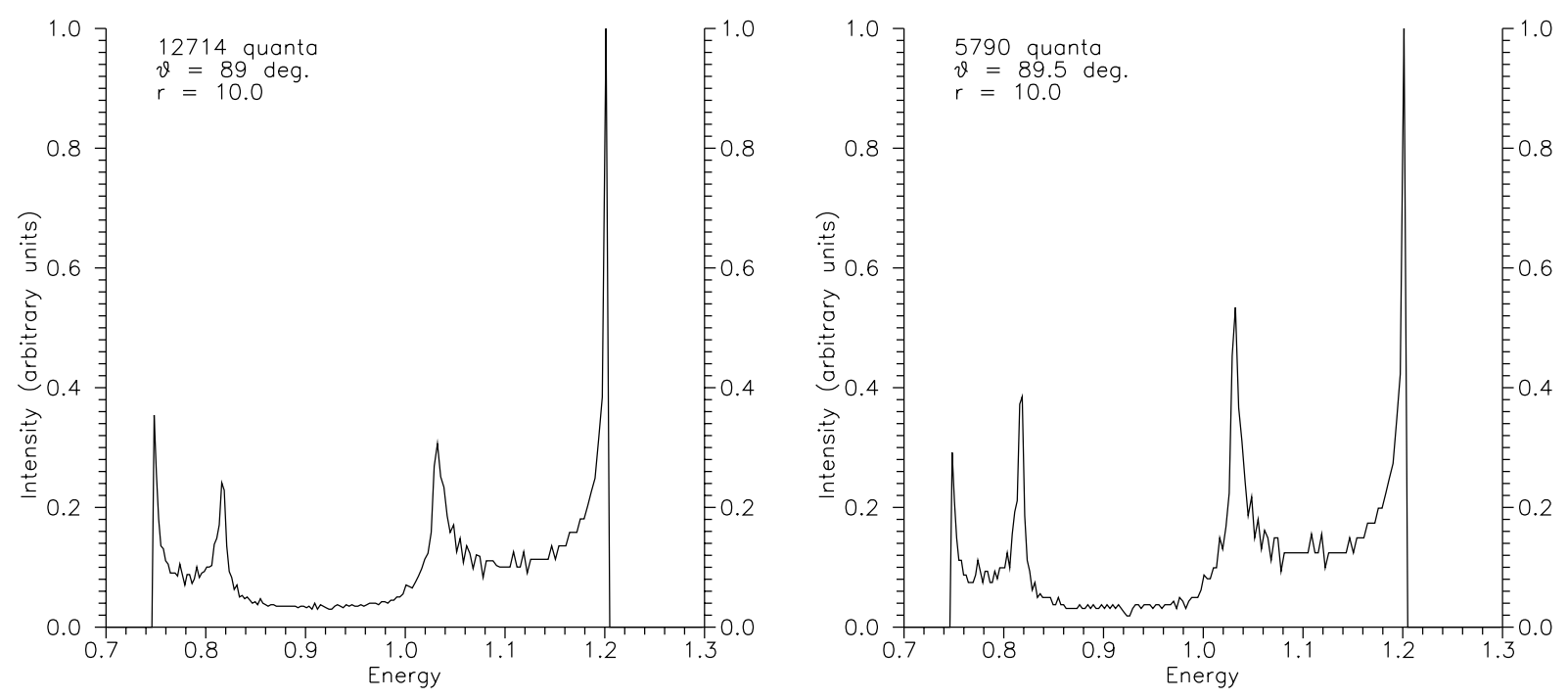

Fig. 2. Details of a hot spot line profile for the most distinctive case with $r=10 r_{\mathrm{g}}$ and $a=0.9981$ (see the top row of Fig. 1). The images of all orders are counted. The left panel includes all the quanta, registered at infinity with $\theta>89^{\circ}$. The right panel includes the quanta with $\theta>89.5$.
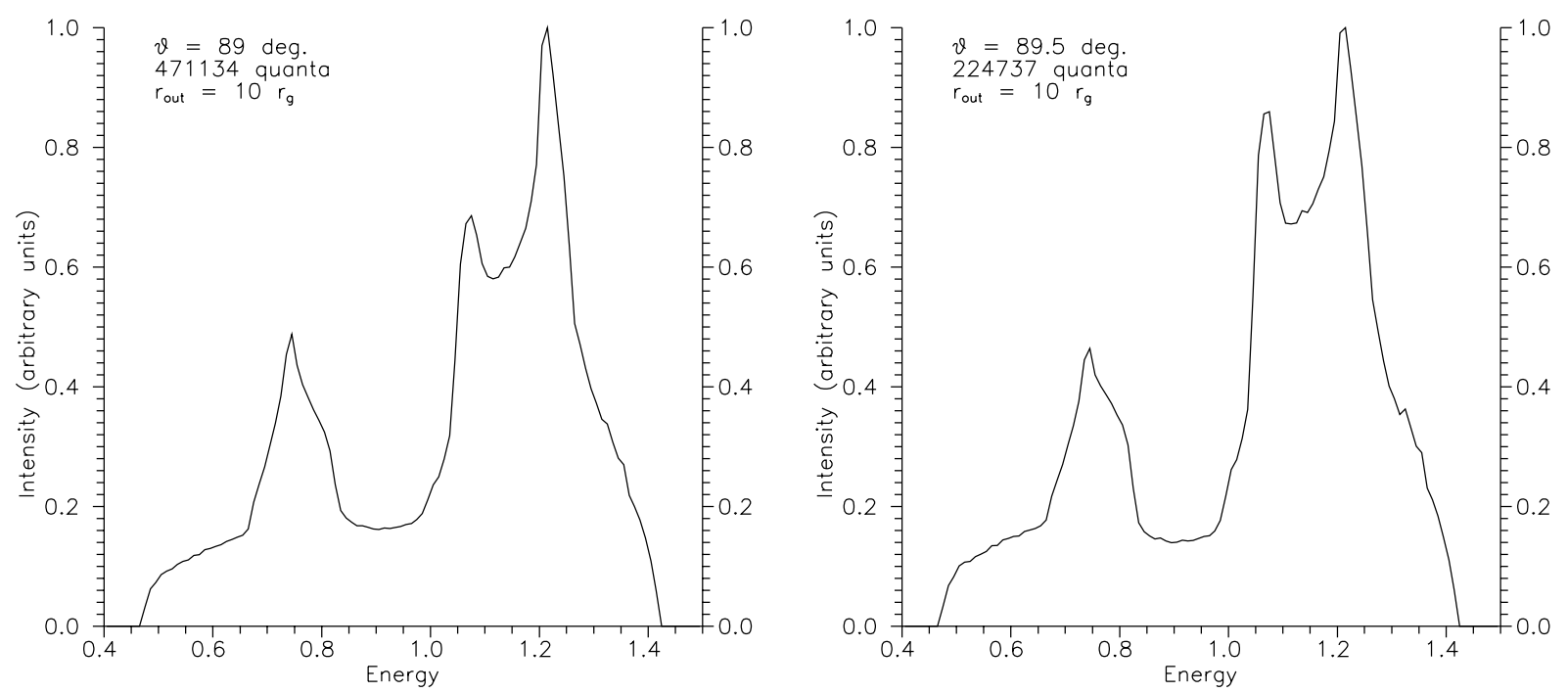

Fig. 3. Details of the line structure for an $\alpha$-disk in the Schwarzschild metric with outer and inner edges of emitting region equal to $r_{\text {out }}=10 r_{\mathrm{g}}$ and $r_{\text {in }}=3 r_{\mathrm{g}}$, respectively. The left panel includes all the quanta, registered at infinity with $\theta>89^{\circ}$. The right panel includes the quanta with $\theta>89^{\circ} .5$.

Bao (1993). The authors argued that there are non-negligible chances to observe such phenomenon in some AGNs and X-ray binary systems. For example, Bao (1993) suggested that NGC 6814 could be a candidate to demonstrate such a phenomenon (but Madejski et al. 1993 found that the peculiar properties of NGC 6814 are caused by a cataclysmic variable like an AM Herculis System).

However, it is clear that in general the probability to observe objects where one could find such features of spectral lines is small. Moreover, even if the inclination angle is very close to $90^{\circ}$, the thickness of the disk (shield or a torus around an accretion disk) may not allow us to look at the inner part of accretion disk. Here, discuss astrophysical situations when the the inclination angle of the accretion disk is high enough.

About $1 \%$ of all AGN or microquasar systems could have an inclination angle of the accretion disk $>89^{\circ}$. For example,
Kormendy et al. (1996) found that NGC 3115 has a very high inclination angle about of $81^{\circ}$ (Kormendy \& Richstone 1992 discovered a massive dark object $M \sim 10^{9} M_{\odot}$ (probably a massive black hole) in NGC 3115). Perhaps we have a much higher probability to observe such a phenomenon in X-ray binary systems where black holes with stellar masses could be. Taking into account the precession which is actually observed for some X-ray binary systems (for example, there is a significant precession of the accretion disk for the SS433 binary system (Cherepashchuk 2002) ${ }^{3}$, moreover since the inclination of the orbital plane is high $\left(i \sim 79^{\circ}\right.$ for this object) sometimes we may observe almost edge-on accretion disks of such objects. Observations indicated that there is a strong evidence that

\footnotetext{
${ }^{3}$ Shakura (1972) predicted that if the plane of an accretion disk is tilted relative to the orbital plane of a binary system, the disk can precess.
} 
the optically bright accretion disk in SS433 is in a supercritical regime of accretion. The first description of a supercritical accretion disk was given by Shakura \& Sunyaev (1973). Even now such a model is discussed to explain observational data for SS433 (Cherepashchuk 2002). We used the temperature distribution from the Shakura-Sunyaev model (Shakura \& Sunyaev 1973; Lipunova \& Shakura 2002) for the inner part of accretion disk to simulate shapes of lines which could be emitted from this region (Fig. 3). Therefore, we should conclude that the properties of spectral line shapes discovered by Matt et al. (1993) are confirmed also for such emissivity (temperature) distributions which correspond to the ShakuraSunyaev model.

Thus, such properties of spectral line shapes are robust enough with respect to wide variations of rotational parameters of black holes and the surface emissivity of accretion disks as it was predicted by Matt et al. (1993). In this work their conjecture was confirmed not only for the Kerr black hole case but also for other dependences of surface emissivity of the accretion disk.

Acknowledgements. A.F.Z. is grateful to E. F. Zakharova for the kindness and support necessary to complete this work. AFZ would like to thank Dipartimento di Fisica Universita di Lecce, INFN, Sezione di Lecce and the National Astronomical Observatories of the Chinese Academy of Sciences for a hospitality and profs. F. DePaolis, G. Ingrosso, J. Wang and Dr. Z. Ma for very useful discussions.

S.V.R. is very grateful to Prof. E. Starostenko, Dr. A. Salpagarov and Dr. O. Sumenkova for the possibility of working on this problem.

This work was supported by the National Natural Science Foundation of China, No. 10233050. This work has been partly supported also by the Russian Foundation for Basic Research, grant 00-02-16108.

The authors thank the anonymous referee for useful remarks.

\section{References}

Bao, G. 1993, ApJ, 409, L41

Bao, G., Hadrava, P., \& Ostgaard, E. 1994, ApJ, 435, 55

Bao, G., \& Stuchlik, Z. 1992, ApJ, 400, 163

Bao, G., Hadrava, P., \& Ostgaard, E. 1996, ApJ, 464, 684

Bromley, B. C., Chen, K., \& Miller, W. A. 1997, ApJ, 475, 57

Carter, B. 1968, Phys. Rev. D, 174, 1559

Chandrasekhar, S. 1983, Mathematical Theory of Black Holes (Oxford: Clarendon Press)

Chen, K., Halpern, J. P., \& Filippenko, A. V. 1998, ApJ, 344, 115

Cherepashchuk, A. M. 2002, Space Sci. Rev., 102, 23

Cui, W., Zhang, S. N., \& Chen, W. 1998, ApJ, 492, L53

Cunningham, C. T., \& Bardeen, J. M. 1973, ApJ, 183, 237

Cunningham, C. T. 1975, ApJ, 202, 788

Dumont, A. M., \& Collin-Souffrin, S. 1990, A\&AS, 83, 71

Fabian, A. C., Rees, M., Stella, L., \& White, N. E. 1989, MNRAS, 238, 729

Fabian, A. C., Nandra, K., Reynolds, C. S., et al. 1995, MNRAS, 277, L11

Fabian, A. C., Iwazawa, K., Reynolds, C. S., \& Young, A. J. 2000, PASP, 112, 1145

Fanton, C., Calvani, M., de Felice, F., \& Cadez, A. 1997, PASJ, 49, 159
Gear, C. W. 1971, Numerical Initial Value Problems in Ordinary Differential Equations (Englewood Cliffs, NY: Prentice Hall)

Greiner, J. 2000, in Cosmic Explosions, Proc. of 10th Annual Astrophysical Conf. in Maryland, ed. S. Holt, \& W. W. Zang, AIP Conf. Proc., 522, 307 [astro-ph/9912326]

Hiebert, K. L., \& Shampine, L. F. 1980, Implicitly Defined Output Points for Solutions of ODE-s. Sandia report sand80-0180

Hindmarsh, A. C. 1983, ODEpack, a systematized collection of ODE solvers, in Scientific Computing, ed. R. S. Stepleman (Amsterdam: North-Holland), 55

Karas, V., Vokrouhlický, D., \& Polnarev, A. G. 1992, MNRAS, 259, 569

Kojima, Y. 1991, MNRAS, 250, 629

Kormendy, J., \& Richstone, D. 1992, ApJ, 393, 559

Kormendy, J., Bender, R., Richstone, D., et al. 1996, ApJ, 459, L57

Landau, L. D., \& Lifshitz, E. M. 1971, The classical theory of fields (Oxford: Pergamon)

Laor, A. 1991, ApJ, 376, 90

Lipunova, G. V., \& Shakura, N. I. 2002, Astron. Rep., 46, 366

Ma, Zhen-Guo 2002, Chin Phys. Lett., 19, 1537

Madejski, G. M., Done, C., Turner, T. J., et al. 1993, Nature, 365, 626

Malizia, A., Bassani, L., Stephen, J. B., et al. 1997, ApJSS, 113, 311

Martocchia, A., \& Matt, G. 1996, MNRAS, 282, L53

Martocchia, A., Karas, V., \& Matt, G. 2000, MNRAS, 312, 817

Martocchia, A., Matt, G., \& Karas, V. 2002, A\&A, 383, L23

Martocchia, A., Matt, G., \& Karas, V., et al. 2002, A\&A, 387, 215

Matt, G., Fabian, A. C., \& Ross, R. R. 1993, MNRAS, 262, 179

Matt, G., Perola, G. C., \& Piro, L. 1991, A\&A, 267, 643

Matt, G., \& Perola, G. C. 1992, MNRAS, 259, 63

Matt, G., Perola, G. C., Piro, L., \& Stella, L. 1992a, A\&A, 257, 63

Matt, G., Perola, G. C., Piro, L., \& Stella, L. 1992b, A\&A, 263, 453

Matt, G, Perola, G. C., \& Stella, L. 1993, A\&A, 267, 643

Miller, J. M., Fabian, A. C., Wijnands, R., et al. 2002, ApJ, 570, L69

Mirabel, I. F. 2001, Ap\&SS, 276, 319

Mirabel, I. F., \& Rodriguez, L. F. 2002, Sky \& Telescope, May, 33

Misner, C. W., Thorne, K. S., \& Wheeler, J. A. 1973, Gravitation (San Francisco: W. H. Freeman and Company)

Nandra, K., George, I. M., Mushotzky, R. F., et al. 1997a, ApJ, 476, 70

Nandra, K., George, I. M., Mushotzky, R. F., et al. 1997b, ApJ, 477, 602

Novikov, I. D., \& Thorne, K. S. 1973, in Black Holes, ed. C. De Witt, \& B. S. DeWitt (New York: Gordon \& Breach), 334

Ogle, P. M., Marshall, H. L., Lee, J. C., et al. 2000, ApJ, 545, L81

Pariev, V. I., \& Bromley, B. C. 1997, Line Emission from an Accretion Disk around Black Hole: Effects of the Disk Structure, in Proc. of the 8th Annual October Astrophysics Conference in Maryland Accretion Processes in Astrophysical Systems: Some Like it Hot! College Park, MD, October 1997, ed. S. S. Holt, \& T. R. Kallman, AIP Conf. Proc. 431, 273 [astro-ph/9711214]

Pariev, V. I., \& Bromley, B. C. 1998, ApJ, 508, 590

Pariev, V. I., Bromley, B. C., \& Miller, W. A. 2001, ApJ, 547, 649 [astro-ph/0010318]

Paul, B., Agrawal, P. C., Rao, A. R., et al. 1998, ApJ, 492, L63

Petzold, L. R. 1983, SIAM J. Sci. Stat. Comput., 4, 136

Rauch, K. P., \& Blandford, R. D. 1993, Caltech Preprint GRP-334

Rauch, K. P., \& Blandford, R. D. 1994, ApJ, 421, 46

Robinson, A., Perez, E., \& Binette, L. 1990, MNRAS, 246, 349

Romanova, M. M., Ustyugova, G. V., Koldoba, A. V., et al. 1998, ApJ, 500,703

Ruszkowski, M. 2000, MNRAS, 315, 1

Sambruna, R. M., George, I. M., Mushotsky, R. F., et al. 1998, ApJ, 495,749 
Shakura, N. I. 1972, AZh, 49, 921

Shakura, N. I., \& Sunyaev, R. A. 1973, A\&A, 24, 337

Sharp, N. A. 1981, J. Comput. Phys., 41, 295

Sulentic, J. W., Marziani, P., Zwitter, T., et al. 1998a, ApJ, 501, 54

Sulentic, J. W., Marziani, P., \& Calvani, M. 1998b, ApJ, 497, L65

Tanaka, Y., Nandra, K., Fabian, A. C., et al. 1995, Nature, 375, 659

Weawer, K. A., Krolik, J. H., \& Pier, E. A. 1998, ApJ, 498, 213

Yaqoob, T., Serlemitsos, P. J., Turner, T. J., et al. 1996, ApJ, 470, L27

Yaqoob, T., McKernan, B., Ptak, A., et al. 1997, ApJ, 490, L25

Yaqoob, T., George, I. M., Nandra, K., et al. 2001, ApJ, 546, 759

Zakharov, A. F. 1986, Sov. Phys. - Journ. Experim. and Theor. Phys., 64,1

Zakharov, A. F. 1989, Sov. Phys. - Journ. Experimental and Theoretical Phys., 68, 217

Zakharov, A. F. 1991, SvA, 35, 147
Zakharov, A. F. 1993, Preprint MPA 755

Zakharov, A. F. 1994, MNRAS, 269, 283

Zakharov, A. F. 1995, On the Hot Spot near a Kerr Black Hole, In 17th Texas Symposium on Relativistic Astrophysics, Ann. NY Academy of Sciences, 1995, 759, 550

Zakharov, A. F., \& Repin, S. V. 1999, Astron. Rep., 43, 705

Zakharov, A. F., \& Repin, S. V. 2002a, Astron. Rep., 46, 360

Zakharov, A. F., \& Repin, S. V. 2002b, in Proc. of the Eleven Workshop on General Relativity and Gravitation in Japan, ed. J. Koga, T. Nakamura, K. Maeda, \& K. Tomita (Tokyo: Waseda University), 68

Zakharov, A. F., Kardashev, N. S., Lukash, V. N., \& Repin, S. V. 2002, MNRAS, accepted [astro-ph/0212008]

Zakharov, A. F., \& Repin, S. V. 2003, Adv. in Space Res., accepted 